\title{
Theoretical Analysis of a New Technique for Inertial Rotation Sensing Using a Semiconductor Ring Laser
}

\author{
Antonio Pérez-Serrano and Alessandro Scirè
}

\begin{abstract}
We introduce a new technique for the measurement of inertial rotations using a semiconductor ring laser. Inertial rotation introduces a frequency deviation of the optical frequency of the two counterpropagating waves in the laser cavity, whereas mode coupling causes frequency attraction (frequency pushing) and finally locking at low rotation rates, washing out the small Sagnac frequency difference to be measured. Here we propose to measure inertial rotation whitin the so-called locking band using the phase/amplitude dependence on detuning found for the oscillating modes under the gain line. The dephasing accumulated by the two counterpropagating waves unbalances the fields' amplitudes within the locking region. We analytically derive the responsivity function quantifying the two-mode power unbalance versus rotation rate, by means of a two-mode rate equations model. Noise performance of a possible rotations sensor are also discussed by calculating the noise equivalent rotation rate.
\end{abstract}

Index Terms-Laser dynamics, metrological applications, ring lasers, semiconductor lasers.

\section{INTRODUCTION}

N EMICONDUCTOR ring lasers (SRLs) [1] raised interest because their monolithic integration is easily achievable [2]. SRLs are used in numerous applications like wavelength filtering, unidirectional travelling-wave operation [3], multiplexing/demultiplexing applications, and data storage-gating as optical bistables [4]-[6]. One interesting potential application of an SRL is a semiconductor ring laser gyroscope (S-RLG) [7], [8], which could represent a compact, simple, and low-cost optical device for inertial rotation sensing.

The idea of using a ring interferometer as a rotation rate sensor was introduced by Sagnac [9], and became a technical reality after the assessment of a reliable laser technology. Optical gyroscopes are currently used in satellites, aircrafts, and remote-control devices. RLGs are based on the measurement of the detuning induced by rotation onto the optical frequency of the two counterpropagating modes in a ring laser. However, any source of intracavity backscattering couples and can lock the counterpropagating waves [10]-[12], representing a major source of error in practical devices [13].

The advantages of an S-RLG are monolithic integration, potential low cost, and small size. However, integrated S-RLGs

Manuscript received December 11, 2008; revised March 11, 2009. First published April 10, 2009; current version published June 17, 2009. This work was supported by the European Union project IOLOS-FP6-IST-2005-34743, by the Spanish Government project PhoDeCC TEC2006-10009/MIC, and by the Balear Government project QULMI PROGECIB-5A. The work of A. Scirè was supported by the Ramón y Cajal program by Spanish MEC.

The authors are with the Instituto de Física Interdisciplinar y Sistemas Complejos, IFISC, CSIC-UIB, E-07071 Palma de Mallorca, Spain (e-mail: antonio@ifisc.uib.es; scire@ifisc.uib.es; http://ifisc.uib.es).

Digital Object Identifier 10.1109/LPT.2009.2019622 show a wide locking band of the order of 10-100 MHz [14] hindering the Sagnac effect for practical rotation rates.

In this letter, we theoretically show that an SRL can be used to measure inertial rotation within the so-called locking band, i.e., without the need to unlock the two counterpropagating waves. Indeed, the dephasing accumulated by the two counterpropagating waves due to rotation within the locking region is coupled to the field amplitudes via conservative backscattering. This in turn unbalances the field amplitudes by a quantity proportional to the rotation angular velocity. We provide analytical expression for the responsivity that would characterize a possible rotation sensor. Moreover, we consider the quantum fluctuations [8] of the fields to calculate the noise equivalent rotation rate (NER), obtaining $10^{-3} \mathrm{~Hz}$ assuming $10 \mathrm{~mW}$ of output power. The obtained NER is higher than what is tipically displayed by $\mathrm{He}-\mathrm{Ne}$ RLG; however, the limited cost and size of an S-RLG could make it appealing for rotation sensing applications. Also, this technique can be exported to other ring lasers than SRLs, if an intracavity mechanism of conservative backscattering is provided.

The theoretical analysis is based on a set of dimensionless dynamical equations [14], [15] for the two (slowly varying) complex amplitudes of the counterpropagating fields $E_{+}$and $E_{-}$ modified to account for inertial rotation effects [10], [16] and a Bloch equation for the carrier density $N$. The equations read

$$
\begin{aligned}
\dot{E}_{ \pm}(t) & =\mathcal{G}_{ \pm}\left(N(t),\left|E_{ \pm}(t)\right|^{2}\right) E_{ \pm}(t)-\eta E_{\mp}(t) \pm i \Delta E_{ \pm}(t) \\
\dot{N}(t) & =\gamma \mathcal{F}\left(N(t),\left|E_{ \pm}(t)\right|^{2}\right)
\end{aligned}
$$

where

$$
\begin{aligned}
\mathcal{G}_{ \pm}\left(N(t),\left|E_{ \pm}(t)\right|^{2}\right)= & \frac{1}{2}(1+i \alpha)\left\{N(t) \sigma_{ \pm}-1\right\} \\
\mathcal{F}\left(N(t),\left|E_{ \pm}(t)\right|^{2}\right)= & \mu-N(t)-N(t) \sigma_{+}\left|E_{+}(t)\right|^{2} \\
& -N(t) \sigma_{-}\left|E_{-}(t)\right|^{2} \\
\sigma_{ \pm}= & 1-s\left|E_{ \pm}(t)\right|^{2}-c\left|E_{\mp}(t)\right|^{2} .
\end{aligned}
$$

The parameter $\alpha$ accounts for phase-amplitude coupling and the self and cross saturation coefficients are given by $s$ and $c$, respectively; $\eta=k_{d}+i k_{c}$ is the complex backscattering coefficient where the parameters $k_{d}$ and $k_{c}$ represent the dissipative and conservative components of the backscattering, respectively, and $2 \Delta$ is the rotation-induced frequency difference. The carrier density $N$ obeys the Bloch equation for semiconductor lasers, where $\mu$ is the dimensionless pump ( $\mu=1$ at laser threshold). In the set (1), the dimensionless time is rescaled by the photon lifetime $\tau_{p}$. The parameter $\gamma$ is the ratio of the carrier lifetime $\tau_{s}$ over $\tau_{p}$

Consistently with the standard theory [16], the emission frequency of the two modes (referred to a common optical carrier 
set to zero) is shifted by the inertial rotation of a dimensionless (because of time rescaling) amount equal to $2 \Delta$, when the rotation vector is orthogonal to the cavity plane

$$
\Delta=\frac{2 \pi R \tau_{p}}{\lambda} \Omega_{\mathrm{rot}}
$$

where $R$ is the ring radius, $\lambda$ is the laser wavelength, and $\Omega_{\text {rot }}$ is the dimensional rotation angular velocity. First we analyze the SRL at rest $(\Delta=0)$. We look for a solution of the following form for the set (1):

$$
E_{ \pm}(t)=Q_{ \pm} e^{i(\omega t \pm \psi / 2)}, \quad N(t)=\bar{N}
$$

By substitution of (3) in the set (1) with $\Delta=0$, we find [15] symmetric steady-state solutions for the carrier density and the amplitudes of the fields

$$
\begin{aligned}
\bar{N} & =\frac{\mu}{1+2 Q^{2}-2(c+s) Q^{4}} \\
Q_{ \pm}^{2} & =Q^{2}=\frac{\bar{N}-1+k_{d}}{(c+s) \bar{N}} .
\end{aligned}
$$

There are two possible cases characterized by two stationary values of the relative phase, the in phase case, $\psi=0$, with corresponding oscillation frequency

$$
\omega_{i n}=\alpha k_{d}-k_{c}
$$

and the out of phase case, $\psi=\pi$, with corresponding frequency

$$
\omega_{\text {out }}=-\alpha k_{d}+k_{c} \text {. }
$$

Depending on the sign of the backscattering parameters, one of the solutions is stable and the other unstable; if $k_{d}>0$ the out of phase case is stable, and the in phase case is stable for $k_{d}<0$. The set (1) presents other solutions (i.e., alternate oscillations, bistability); in this work, we focus on the effect of a Sagnac detuning on the symmetric solution. From now on, we focus on the in phase case without loosing generality. If $\Delta$ is small, the stationary solution deviate from the solution at rest (4)-(6). Assuming a amplitude deviation of the form

$$
Q_{ \pm}=Q \pm \delta
$$

and a small deviation $\theta$ of the relative phase $\psi$. By substituting the deviations with (3) in the set (1), at first order in $\delta$ and $\theta$, separating the real and the imaginary part, we obtain a set of two linear coupled equations for the perturbations $\delta$ and $\theta$, i.e.,

$$
\begin{aligned}
& \Delta Q+k_{d} \theta Q+2 k_{c} \delta-\alpha Q^{2} \delta \bar{N}(s-c)=0 \\
& 2 k_{d} \delta-k_{c} \theta Q-\bar{N} Q^{2} \delta(s-c)=0 .
\end{aligned}
$$

We define $\chi$ as the difference between the fields' intensities divided by the total intensity; using (3) with (4)-(8), we obtain

$$
\chi=\frac{\left|E_{-}\right|^{2}-\left|E_{+}\right|^{2}}{\left|E_{+}\right|^{2}+\left|E_{-}\right|^{2}}=\frac{2}{Q} \delta=\mathcal{R} \Omega_{\mathrm{rot}}
$$

where $\mathcal{R}$ is the responsivity of the system to the dimensional inertial rotation $\Omega_{\text {rot }}$; by solving (9) and using (10), we find the analytical expression for the dimensional $\left[\mathrm{Hz}^{-1}\right]$ responsivity function

$$
\mathcal{R}=\frac{4 \pi k_{c} R \tau_{p}}{\lambda\left(2 k_{d}^{2}+2 k_{c}^{2}-\left(k_{d}+\alpha k_{c}\right) Q^{2} \bar{N}(s-c)\right)} .
$$

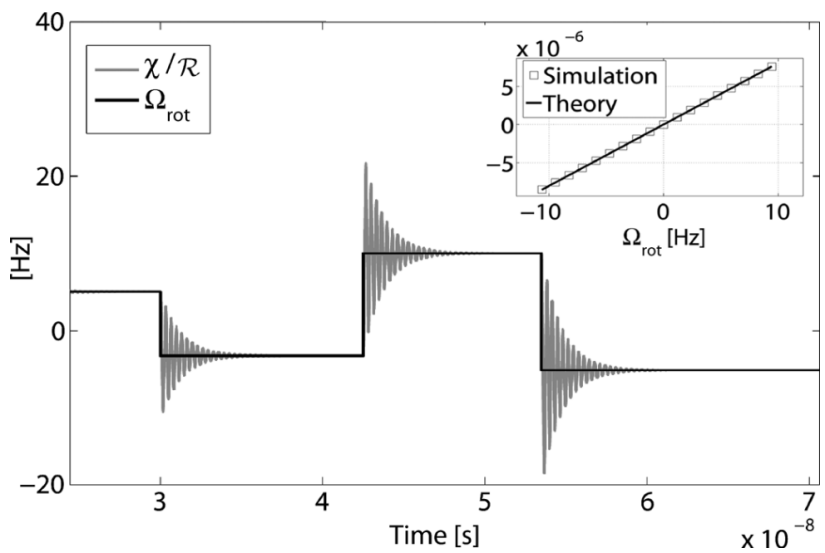

Fig. 1. Numerical simulations of the response of the SRL to a rotation depending on time. Inset: System response $\chi$ versus inertial rotation $\Omega_{\text {rot }}$. The slope is the responsivity $\mathcal{R}$. The ring radius is $R=600 \mu \mathrm{m}$ and the pump is $\mu=1.2, \alpha=3.5, s=0.005, c=0.01, k_{d}=-3.2710^{-4}, k_{c}=4.410^{-3}$, and $\tau_{p}=1 \mathrm{ps}$. The parameter set is taken according to experimental fitting [15].
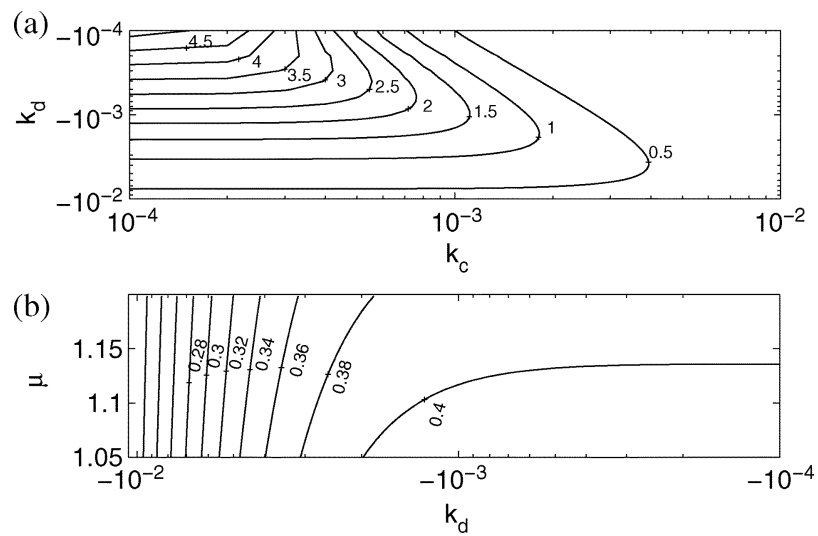

Fig. 2. (a) Responsivity $\left(x 10^{-5}\right)$ contour plot versus backscattering coefficients, $\mu=1.2$; (b) responsivity $\left(x 10^{-5}\right)$ contour plot versus backscattering coefficient $k_{d}$ and pump current, $k_{c}=10^{-2} . \alpha=3.5, s=0.005, c=0.01$, $\tau_{p}=1 \mathrm{ps}, R=600 \mu \mathrm{m}$, and $\lambda=890 \mathrm{~nm}$.

The responsivity $\mathcal{R}$ quantifies how the rotation unbalances the counterpropagating fields' intensities. In Fig. 1 (inset), $\chi$ is plotted versus the inertial rotation $\Omega_{\text {rot }}$ using the analytical expression (10), and compared to numerical simulations. Fig. 1 (inset) shows that our analytical approximations are well met and how rotation can be resolved without sign ambiguity.

Fig. 2(a) shows the responsivity behavior versus the real and imaginary part of the backscattering coefficient. Fig. 2(b) shows responsivity versus backscattering dissipative coefficient $k_{d}$ and pump current $\mu$. As a general trend, the responsivity decreases for increasing values for the dissipative backscattering coefficient and decreasing values for the conservative backscattering coefficient. Physically, the dissipative backscattering stabilizes the locking by damping the perturbations of the phase difference between the two modes. On the other side, conservative backscattering enhances the relative phase dynamics, thus making the system more sensitive to sources of dephasing like the Sagnac effect. Indeed (11) shows that the responsivity vanishes if $k_{c}=0$. This is so because the relative dephasing accumulated by the two fields is coupled to the field amplitudes via conservative backscattering when $\Omega_{\text {rot }}$ is within the locking band. This effect is different from the amplitude 

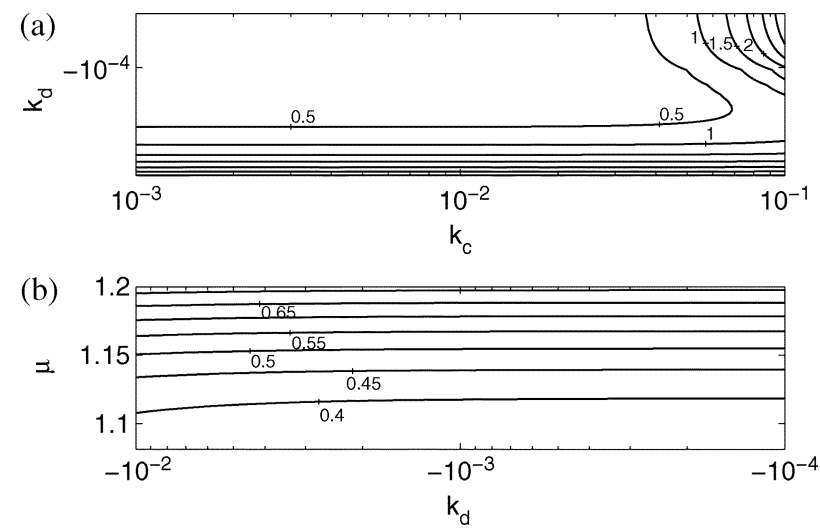

Fig. 3. (a) $\Omega_{\mathrm{NER}}\left(x 10^{-2}\right)$ contour plot versus backscattering coefficients, $\mu=1.2$; (b) $\Omega_{\mathrm{NER}}\left(x 10^{-2}\right)$ contour plot versus backscattering coefficient $k_{d}$ and pump current $\mu, k_{c}=10^{-2} . \alpha=3.5, s=0.005, c=0.01, \tau_{p}=1 \mathrm{ps}$, $R=600 \mu \mathrm{m}, \lambda=890 \mathrm{~nm}, B=10 \mathrm{~Hz}$, and $P=10 \mathrm{~mW}$.

modulation reported in [17], because in our case the two fields unbalance their continuos-wave component.

To characterize the response of the device to a rotation variation, we simulate the equation set (1) with a time-dependent rotation. The results are shown in Fig. 1; the response time of the device is a few nanoseconds for our parameter's choice.

Noise characteristics are also important to characterize the possible implementation of this technique in real gyroscopes. Considering the quantum fluctuations [8] for the optical power in each direction $P_{ \pm}=\left|E_{ \pm}\right|^{2}$

$$
\left\langle\delta P_{ \pm}^{2}\right\rangle=\frac{2 h c B P_{ \pm}}{\lambda}
$$

where $B$ is the instrument bandwidth, $c$ is the speed of light, and $h$ is Planck's constant. Using (12) with (10), assuming $P_{+}=$ $P_{-}=P$ by straightforward calculation, we obtain the standard deviation of $\chi$, assuming $\left\langle\delta P_{ \pm}^{2}\right\rangle^{1 / 2} \ll P$

$$
\sigma_{\chi}=\left\langle\delta \chi^{2}\right\rangle^{1 / 2} \simeq \sqrt{\frac{h B c}{\lambda P}}=\mathcal{R} \Omega_{\mathrm{NER}}
$$

that permits to calculate the noise equivalent rotation $\Omega_{\mathrm{NER}}$. Fig. 3(a) shows $\Omega_{\mathrm{NER}}$ versus backscattering coefficients. The order of $\Omega_{\mathrm{NER}}$ is $\sim 10^{-3} \mathrm{~Hz}$ for a wide range of parameter values for backscattering coefficients. $\Omega_{\mathrm{NER}}$ grows with $k_{c}$ and decreases with $k_{d}$. This is so because the conservative backscattering increases the phase-noise [18], whereas the dissipative backscattering dumps phase fluctuations. Fig. 3(b) shows how $\Omega_{\text {NER }}$ grows with the pump current.

\section{CONCLUSION}

We have introduced a simple theory for a new technique to measure inertial rotation using an SRL. We have derived an analytical expression for the responsivity function of a possible rotation sensor using a well-established two-mode rate equation model. We have investigated the effect of the backscattering coefficients and the pump current on the responsivity function, and the dynamic response of the device when a time-dependent rotation rate is applied. We have considered quantum fluctuations in order to calculate the NER. Our conclusion is that the proposal of using an SRL as a rotation sensor is viable, as it is not necessarily limited by locking effects; the responsivity and noise performance are quite interesting compared to commercial laser gyroscope, taking into account the cost and size benefits of semiconductor laser technology. Moreover, this technique can be exported to any ring-laser gyroscope, providing a intracavity mechanism of conservative backscattering.

\section{ACKNOWLEDGMENT}

The authors would like to thank R. Zambrini for helpful comments.

\section{REFERENCES}

[1] A. S. H. Liao and S. Wang, "Semiconductor injetion lasers with circular resonator," Appl. Phys. Lett., vol. 36, no. 10, pp. 801-803, May 1980.

[2] T. Krauss, P. J. R. Laybourn, and J. S. Roberts, " $\mathrm{CW}$ operation of semiconductor ring lasers," Electron. Lett., vol. 26, pp. 2095-2097, Dec. 1990.

[3] J. J. Liang, S. T. Lau, M. H. Leary, and J. M. Ballantyne, "Unidirectional operation of waveguide diode ring lasers," Appl. Phys. Lett., vol. 70, pp. 1192-1194, Mar. 1997.

[4] M. T. Hill, H. J. S. Dorren, T. de Vrie, X. J. M. Leijtens, J. H. den Besten, B. Smalbrugge, Y. S. Oei, H. Binsma, G. D. Khoe, and M. K. Smit, "A fast low-power optical memory based on coupled micro-ring laser," Nature, vol. 432, p. 206, Nov. 2004.

[5] M. Sorel, G. Giuliani, S. Donati, and P. J. R. Laybourn, "Unidirectional bistability in semiconductor waveguide ring lasers," Appl. Phys. Lett., vol. 80, pp. 3051-3053, Apr. 2000.

[6] T. Pérez, A. Scirè, G. Van der Sande, P. Colet, and C. R. Mirasso, "Bistability and all-optical switching in semiconductor ring lasers," Opt. Express, vol. 15, pp. 12941-12948, Oct. 2007.

[7] P. J. R. Laybourn, M. Sorel, G. Giuliani, and S. Donati, "Integrated semiconductor laser rotation sensors," in Proc. SPIE Conf. Integrated Optics Devices III, 1999, vol. 3620, pp. 322-331.

[8] S. Donati, Electro-Optical Instrumentation: Sensing and Measuring with Lasers. Englewood Cliffs, NJ: Prentice-Hall, 2004.

[9] G. Sagnac, "L'ether lumineux demontre par l'effect du vent relatif d'ether dans un interferometre en rotation uniforme," C.R. Acad. Sci., vol. 157 , p. 708, 1913.

[10] R. J. C. Spreeuw, R. C. Neelen, N. J. van Druten, E. R. Eliel, and J. P. Woerdman, "Mode coupling in a He-Ne ring laser with backscattering," Phys. Rev. A, vol. 42, pp. 4315-4324, Oct. 1990.

[11] C. Etrich, P. Mandel, R. C. Neelen, R. L. C. Spreew, and J. P. Woerdman, "Dynamics of a ring-laser gyroscope with backscattering," Phys. Rev. A, vol. 46, pp. 525-536, Jul. 1992.

[12] F. C. Cheng, "Investigation a dye ring laser with backscattering," Phys. Rev. A, vol. 45, pp. 5220-5227, Apr. 1992.

[13] W. W. Chow, J. Gea-Banacloche, L. M. Pedrotti, V. E. Sanders, W. Schleich, and M. O. Scully, "The ring laser gyro," Rev. Mod. Phys., vol. 57, no. 1, pp. 61-104, Jan. 1985.

[14] M. Sorel, G. Giuliani, A. Scirè, R. Miglieria, S. Donati, and P. J. R. Laybourn, "Operating regimes of GaAs-AlGaAs semiconductor ring lasers: Experiment and model," IEEE J. Quantum Electron., vol. 39, no. 10, pp. 1187-1195, Oct. 2003.

[15] M. Sorel, A. Scirè, G. Giuliani, R. Miglierina, S. Balle, P. J. R. Laybourn, and S. Donati, "Alternate oscillations in semiconductor ring lasers," Opt. Lett., vol. 27, pp. 1992-1994, 2002.

[16] L. N. Menegozzi and W. E. Lamb, Jr., "Theory of a ring laser," Phys Rev. A, vol. 8, no. 4, pp. 2103-2125, Oct. 1973.

[17] V. Annovazzi-Lodi, S. Donati, and M. Manna, "Chaos and locking in a semiconductor laser due to external injection," IEEE J. Quantum Electron, vol. 30, pp. 1537-1541, Jul. 1994.

[18] A. Pérez-Serrano, R. Zambrini, A. Scirè, and P. Colet, "Noise spectra of a semiconductor ring laser in the bidirectional regime," Phys. Rev. $A$, submitted for publication. 